\title{
Turbulent horizontal convection at high Schmidt numbers
}

\author{
Pierre-Yves Passaggia," Matthew W. Hurley, Brian White, and Alberto Scotti \\ Marine Sciences Department, University of North Carolina, Venable and Murray Hall, \\ Chapel Hill, North Carolina 27599, USA ${ }^{\dagger}$
}

(Received 17 May 2017; published 29 September 2017)

\begin{abstract}
This paper is associated with a poster winner of a 2016 APS/DFD Milton van Dyke Award for work presented at the DFD Gallery of Fluid Motion. The original poster is available from the Gallery of Fluid Motion, https://doi.org/10.1103/APS.DFD.2016.GFM.P0028
\end{abstract}

DOI: 10.1103/PhysRevFluids.2.090506

Horizontal convection is flow driven by a buoyancy gradient imposed along a horizontal boundary. It is a simple model to study the influence of heating, cooling, and fresh water fluxes at the ocean surface on the meridional overturning circulation [1]. In order to investigate the flow properties and energetics of horizontal convection at high Schmidt numbers $\mathrm{Sc}=v / \kappa$, where in the present case $v \approx 10^{6} \mathrm{~m}^{2} / \mathrm{s}$ is the kinematic viscosity and $\kappa \approx 1.64 \times 10^{9} \mathrm{~m}^{2} / \mathrm{s}$ is the diffusion coefficient of salt in water, the flow is driven by the diffusion of salt in water across reinforced permeable

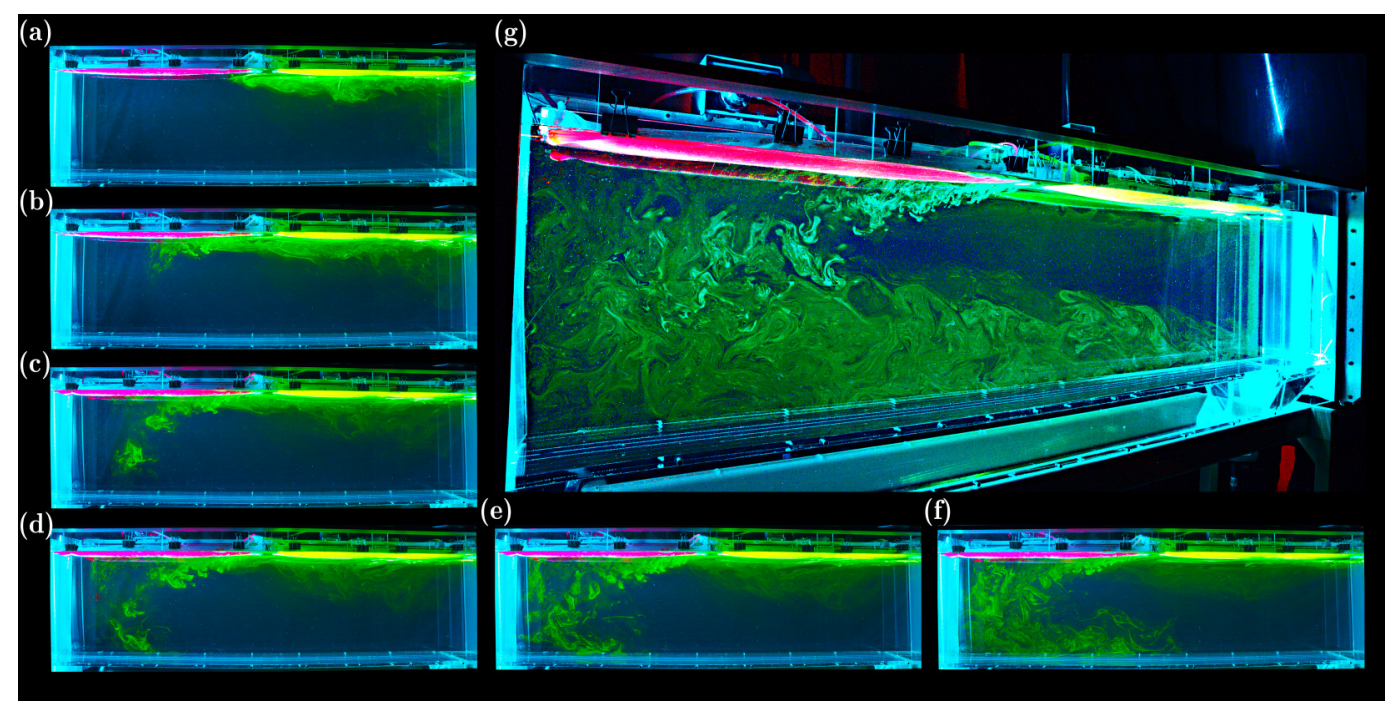

FIG. 1. Side view of the tank, illuminated from the left and showing the evolution of fresh fluorescein dye water released from the right. The temporal evolution of the circulation is described by the transport of the fluorescein (green/yellow) dye, driven by a solutal horizontal density gradient, creating a turbulent plume sinking on the left of the tank (a-f). The deep circulation is shown in $(\mathrm{g})$ where the flow has developed and drives a deep but weakly turbulent circulation that eventually upwells on the right of the tank.

*passaggia@unc.edu

${ }^{\dagger}$ Joint Fluids Lab., University of North Carolina, Chapman Hall, Chapel Hill, North Carolina 27599, USA.

Published by the American Physical Society under the terms of the Creative Commons Attribution 4.0 International license. Further distribution of this work must maintain attribution to the author(s) and the published article's title, journal citation, and DOI. 


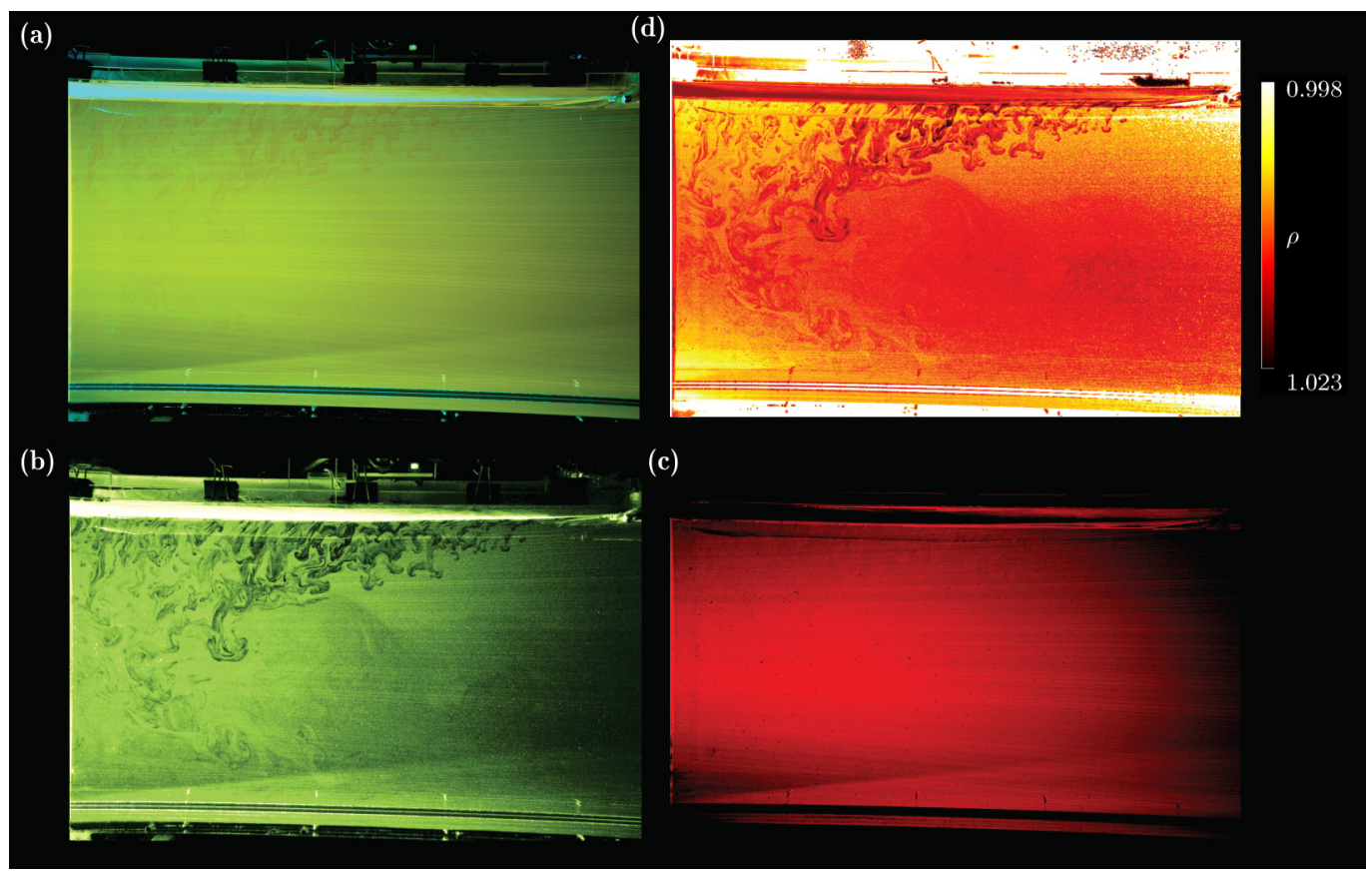

FIG. 2. (a) Raw double emission laser induced fluorescence color image showing the variation of emission of the dye induced by the low concentration of sodium bisulfate, which decreases the $p \mathrm{H}$ of the salty fluid. The images are split in red-green-blue (RGB) channels where the green is associated with the fluorescein dye whose emission decreases with lowering $p \mathrm{H}(\mathrm{b})$. The red channel shows the Rhodamine $\mathrm{WT}$, which is $p \mathrm{H}$ insensitive and shows the distribution of the laser intensity (c). The ratio of dye intensity between (b) and (c) provides an estimate of spatial distribution of the density (d).

Spectra/Por ${ }^{\circledR}$ dialysis membranes with a $12-14 \mathrm{kD}$ porosity. These membranes are stretched over the surface in a $2 \mathrm{~m} \times 0.25 \mathrm{~m} \times 0.5 \mathrm{~m}$ acrylic tank. Two membranes create wells of fresh and salty fluid, both of which are dyed for the visualization of the overturning process. The salty water in the left well is dyed using Rhodamine WT, which appears magenta in color and has an associated density $\left(\rho_{1} \approx 1.023 \mathrm{~g} / \mathrm{cc}\right)$. The right well is filled with fresh water at $\left(\rho_{0} \approx 0.999 \mathrm{~g} / \mathrm{cc}\right)$ and is dyed with fluorescein, which fluoresces yellow/green. The experiment is kept at room temperature $T \approx 22{ }^{\circ} \mathrm{C}$, and the flow is illuminated from the left side using a blue/turquoise laser sheet from an Ar-Ion laser in the range $488-514 \mathrm{~nm}$. A Nikon D4 camera equipped with 24-120 mm Nikkor lens was used to record the flow. The resulting experiments are illustrated for a Schmidt number of $\mathrm{Sc} \approx 610$ and a Rayleigh number $\mathrm{Ra}=\Delta b L^{3} /(v \kappa) \approx 9 \times 10^{14}$ where $L=2 \mathrm{~m}$ is the length of the tank, $\Delta b \approx g\left(\rho_{1}-\rho_{0}\right) / \rho_{0}$ and $g$ is the acceleration of gravity. The visualization is initiated with a few milliliters of fresh water, dyed with fluorescein, injected near the surface on the right side. At early times, the dye travels from the fresh side in a laminar and stratified layer toward the salty side where it sinks as a turbulent plume [see Figs. 1(a)-1(f)] [2]. The bottom of the tank is progressively filled with dyed/salty water which has mixed in the plume and drives a deep circulation [see Fig. 1(g)]. In addition to dye visualizations in Fig. 1, we illustrate the ratiometric Dual Emission Laser Induced Fluorescence (DELIF) technique [3] where the distribution of salinity within the laser sheet can be measured accurately. The spatio-temporal diagnosis of the density of nonhomogeneous salt water is a difficult task, largely because the diffusion coefficient of organic dyes is one order of magnitude lower than that of salt. Instead of using a single dye we introduce a second dye and a blend of sodium bisulfate $\left(\mathrm{NaHSO}_{4}\right)$ with sodium chloride $(\mathrm{NaCl})$ to decrease the $p \mathrm{H}$ of the salt water. The dyes are mixed homogeneously, but the emission of the fluoresecin decreases with decreasing $p \mathrm{H}$ [Fig. 2(b)] 


\section{TURBULENT HORIZONTAL CONVECTION AT HIGH ...}

while Rhodamine WT remains unaffected [Fig. 2(c)]. The ratio between the intensity of the two dies is a visual for the density [Fig. 2(d)] that can be used to measure the local energetics and mixing induced by the overturing circulation in horizontal convection [4].

The authors acknowledge the support by the National Science Foundation under Grant No. NSF-OCE-1155558.

[1] T. Kuhlbrodt, A. Griesel, M. Montoya, A. Levermann, M. Hofmann, and S. Rahmstorf, On the driving processes of the Atlantic meridional overturning circulation, Rev. Geophys. 45, RG2001 (2007).

[2] P.-Y. Passaggia, A. Scotti, and B. White, Transition and turbulence in horizontal convection: linear stability analysis, J. Fluid Mech. 821, 31 (2017).

[3] J. Coppeta and C. Rogers, Dual emission laser induced fluorescence for direct planar scalar behavior measurements, Exp. Fluids 25, 1 (1998).

[4] A. Scotti and B. White, Diagnosing mixing in stratified turbulent flows with a locally defined available potential energy, J. Fluid Mech. 740, 114 (2014). 